\title{
The Technology of Collective Memory and the Normativity of Truth
}

\author{
Kieron O'Hara \\ School of Electronics and Computer Science \\ University of Southampton \\ Highfield \\ Southampton \\ SO17 1BJ \\ kmo@ecs.soton.ac.uk
}

A version of Chapter 18 of David Goldberg, Natasha McCarthy \& Diane Michelfelder (eds), Philosophy and Engineering: Reflections on Practice, Principles and Process, Berlin: Springer-Verlag.

\begin{abstract}
Neither our evolutionary past, nor our pre-literate culture, has prepared humanity for the use of technology to provide records of the past, records which in many context become normative for memory. The demand that memory be true, rather than useful or pleasurable, has changed our social and psychological understanding of ourselves and our fellows. The current vogue for lifelogging, and the rapid proliferation of digital memory-supporting technologies, may accelerate this change, and create dilemmas for policymakers, designers and social thinkers.
\end{abstract}

\section{Introduction}

The relationship between memory, representation and recollection is highly unusual and counterintuitive. In particular, memories can misrepresent past events in what would seem to be all key respects, and yet still facilitate immediate recognition of veridical representations (e.g. video footage of an event). Many psychologists (Loftus and Palmer 1974, Wells 1993), have been able to show that eyewitnesses can be deeply unreliable in recall, especially if misled by queries or interfering information, yet this does not preclude accuracy in identification. The fact that a person was misremembered as having dark hair and a moustache does not mean that they might not be recalled with the shock of recognition: "yes, that's the fellow!" 
Clearly, the 'filing cabinet' metaphor of memory (that it contains a set of representations of the past, organized to facilitate retrieval, such that exposure to a suitable cue will facilitate recall) is as inappropriate as it is naïve (cf. Warnock 1987, 8-9). Memory is constantly changing, in response to conversations with other people about events, constant narration of events by oneself and others, exposure to news reports, photographs, videos, and inference from the effects of the remembered event. My memory of an event may misrepresent the non-moustachioed man, but once I have seen a photograph of him, I realise that he had no moustache, and my memory adapts accordingly.

As the old Maurice Chevalier song had it,

We met at nine.

- We met at eight.

I was on time.

- No, you were late.

Ah yes, I remember it well.

The joke here is that the two singers have completely opposite recollections of the significant event in their lives, and yet agree entirely on its identification. As Marcel Proust (still one of the most acute theorists of memory) argued, one's memories are coloured by one's present assumptions and mental models; an apparently insignificant event can appear significant in retrospect because it contained a first encounter with a person whom one later came to love.

In this chapter I shall discuss the use of technology to support recollection. In particular, one often uses representations such as photographs to support recall. I shall make the obviously idealizing assumption that a photograph does not misrepresent the past in the way that a memory can; the camera was pointed and the image captured. Of course images can be Photoshopped, but that requires human intervention to cause the misrepresentation. Further, images can give a false impression, as for example when a trick of perspective makes a distant large object look near and miniature; again, the misrepresentation requires a human interpreter. As a matter of fact I do not think that mechanical reproductions are essentially veridical representations, but it will make the argument simpler and clearer if we pretend that they are, in contrast with human memories which may or may not be veridical.

I will focus on what is normative for memory, and shall argue that the use of technology has increased the prominence of truth in that role. This is not necessarily a bad thing, but it is a newish development, and as technological supports increase dramatically, will continue to drive important social and psychological change. These considerations should be used to help drive our reactions and regulations in areas such as privacy, deletion, data protection and informational selfdetermination. 


\section{The Technology of Memory}

Human memory has always been a rich source of inspiration and metaphor for computer memory (O'Hara et al 2006a), but our understanding of human, machine and social memory is converging in ways that are more than metaphorical (O'Hara et al 2006b). Memory-supporting technology, which at least initially was conceived as a medical resort, has branched out into the areas of leisure, social networking and self-improvement (Garde-Hansen et al 2009).

Moore's Law have taken such technology out of the medical arena and into the social. The fact that one can more or less store anything one likes means that recording requires a very low cognitive overhead - one needn't worry about the extremely tedious tasks of choosing what information to store, or deciding what to delete when the memory gets full. Meanwhile, improved search and retrieval techniques mean that one can find what one needs relatively straightforwardly. One can, in short, use memory technology indiscriminately - which makes it usable (O'Hara et al 2009).

Furthermore, the indiscriminate use of such technology chimes in with the associative ways that human memory works. We store all sorts of pieces of 'useless' information, precisely because we do not know at storage time what will be useful in the future. The guesses we make about what memories are likely to be important in the future are unlikely to be right all the time, so the more raw material that is present in our records of the past, the more likely we are to have everything that is useful (Bell and Gemmell 2009).

It has been calculated that it would be straightforward to store 70 years of high quality video taken from a lifetime (Dix 2002); this has prompted the United Kingdom Computing Research Committee ${ }^{1}$ to propose 'Memories for Life' as a Grand Challenge for computing research (Shadbolt 2003, O'Hara et al 2006b) - in other words, a potentially epoch-making area for research where breakthroughs would promote not only computer science, but also social well-being in a wide population (http://www.ukcrc.org.uk/grand-challenge/current.cfm). As a Grand Challenge, research groups have been coalescing in this area, looking for example at the use of machines to act as companions for humans (Wilks 2010, O'Hara 2010a), or the difficulties for archivists in curating the digital records of noteworthy people. ${ }^{2}$ Elsewhere, special-purpose tools have been helping communities use websites as collective memory resources. ${ }^{3}$

Prosthetic memory has been a major area of research. For instance, one device, the SenseCam developed by Microsoft, ${ }^{4}$ is a small digital camera designed to take

\footnotetext{
${ }^{1}$ An expert panel of the British Computer Society, the Council of Professors and Heads of Computing, and the Institution of Engineering and Technology to promote computing research in the UK (http://www.ukcrc.org.uk/about/index.cfm).

2 http://www.bl.uk/digital-lives/.

3 See e.g. http://www.bbc.co.uk/dna/memoryshare/ or http://www.livememories.org/Home.aspx.

${ }^{4}$ http://research.microsoft.com/en-us/um/cambridge/projects/sensecam/.
} 
photographs passively, without user intervention, while it is being worn around the neck. It has no viewfinder or display to frame photos, but instead is fitted with a wide-angle lens that maximizes its field-of-view, ensuring that nearly everything in the wearer's view is captured (Hodges et al 2006). To review the SenseCam output, it is remarkably effective to run the resulting set of pictures as a speededup movie (De Bruijn and Spence 2002).

SenseCams have been shown to have remarkable positive effects on the memories of at least some sufferers of severe memory impairment (Berry et al 2007). However, these and similar devices are also used more and more frequently to record the behaviour of those with non-impaired memories, either to achieve an objective picture of real-life behaviour (of great value, for example, in market research - cf. Byrne et al 2008), or simply to record the quotidian details of daily life (Lee et al 2008, Doherty et al 2009).

The practice of using such devices to record daily life in an indiscriminate way is called lifelogging. The lifelogger simply uses devices that amass information, and then stores the results. The SenseCam is a special-purpose recording device, but one can also use devices with other functions that generate records as byproducts; mobile phones, Web browsers, email programs, social networking sites and medical sensors all generate information that is of potential interest to the lifelogger (especially among younger people with their greater tendency to integrate digital and connected technology into their daily lives - O'Hara et al 2009).

There are many important pioneers in this space, including Steve Mann who has for many years worn devices to record his daily life, ${ }^{5}$ and Jennifer Ringley, who achieved notoriety in 1996 for broadcasting the output of a camera in her bedroom across the Web (the so-called JenniCam - Jimroglou 1999). Perhaps the most committed is Microsoft executive Gordon Bell, who has developed a suite of technologies and practices to deal with the giant quantities of information one can generate in a normal life, and who has written about the potentially transformative effects of such technologies for work, health and learning, as well as in everyday life (Bell and Gemmell 2009).

If such technologies become more ubiquitous, then they will have social effects with which we all will have to deal. A lifelogging world would be characterised by universality, both in terms of a high proportion of people owning extensive records of their lives, and of those digital records covering a high proportion of people's activities, so that more people would have access to more of their past lives. Such records are likely to be relatively durable; even though there is always a danger of file formats becoming outdated and unsupported by present-day machines, the greater awareness of this problem in the computing industry means that more adaptable general-purpose standards for representational formats are likely to emerge. There is a strong likelihood that lifelogging records would be shared, not only because of the relative ease of copying and transfer compared to nondigital formats, but also because of a greater willingness to use the World Wide

\footnotetext{
${ }^{5} \mathrm{http}: / /$ www.eecg.toronto.edu/ mann/.
} 
Web as a sharing format, for instance on social networking sites (O'Hara et al 2009). The power of a great deal of information amalgamated from several of one's own devices, the lifelogging stores of others, information from social networks (e.g. Facebook or Flickr) and publicly-available information (e.g. using Google or Wikipedia) could be immense in the provision of a rich picture of one's own life (and, as a by-product, of other people's too).

\section{The Normativity of Truth for Memory}

We (and other animals) have memories because they help the organism survive. Our bodies have mechanisms that allow the world outside to change some of their states, allowing adaptation to, and ultimately recall of significant episodes. There is no need for those episodes to be represented exactly or accurately; it may be that the value of a fear reflex is greater if it is triggered more often than need be (in other words, that the 'memory' of an organism is more effective if it tends to generate falsely positive identifications of threats). Forgetting also has its own adaptive value when the past event was traumatic. Memory's utility stems from the smooth functioning of the self rather than the veridicality of its representations, as the novelist Sebastian Barry suggests:

It wasn't so much the question of whether she had written the truth about herself, or told the truth, or believed what she wrote and said were true, or even whether they were true things in themselves. The important thing seemed to me that the person who wrote and spoke was admirable, living, and complete. (Barry 2008, 309)

The use of external objects and constructed aspects of the environment to support memory is relatively recent and has tended to colour our perceptions of what is important about memory. Studies of oral cultures, which lack recourse to permanent representations, show that memory and the reconstruction of the past can have very different properties than we are used to in our technological world (Goody 1998, Ong 1982, esp.57-67, 95-99, 136-152).

In such cultures, verbatim recall of lists or words is rare - unsurprisingly, as it has very little obvious function in such a society. Early anthropologists occasionally dismissed the memories of 'primitives' as flawed because they had difficulty in regurgitating lists of words - yet of what use is that ability when one has no examinations to pass? Recollection becomes a performance, a creative act. History, for instance, becomes indistinguishable from politics, so that when an elder recites the ancestors of a chief through an implausibly large number of generations, what he is really doing is placing the chief in a political context which makes sense. The 'ancestors' that are mentioned allow connections to be made between important dynasties, and so the elder is not performing an impressive feat of memory, but rather reflecting current power structures. Memory of past events, or of a complex ceremony, is distributed across the participants of the discourse. The aim of mnemonics is to stimulate, not to aid recall. All communication is face-to-face, and so 
there is no need to leave records for others to use in the future, or to 'speak' to people remotely.

In an oral culture, the whole notion of 'misrepresentation' is up for grabs. What is the truth here, when there is no permanent certified 'truth' or record available for comparison? The 'fact' that the chief's great-great-great-great-greatgrandmother is such-and-such will be a matter of the completest indifference to him, and so there will be no attempt to keep any kind of record of it; hence when the elder announces a family connection that everyone accepts, what counts is that it is acceptable.

The development of literacy gradually provided a certified record against which individual memories could be compared for accuracy. Written words supported recall, but also furnished an independent standard. Adjustment to the literate world took time. In Plato's Phaedrus, Socrates took issue with those who relied on the written word; 'You have not discovered a potion for remembering, but for reminding; you provide your students with the appearance of wisdom, not with its reality' (Plato 1997, 552). This attitude remained for centuries; when Montaigne wrote phrases all over the beams in his tower, this was not to remind him of their content but rather to provoke new and interesting thoughts of his own.

With the assistance of technology, writing and later photography evolved from being simply supports of memory. The inheritances of Gutenberg and Daguerre were the fixed objective records that were widely understood and shared through all levels of society. In such an environment, a new aspect of memory became possible. Memory could be held to account against the public record, and could be held as 'wrong' if it contradicted it. Truth became normative for memory.

This, of course, is a caricature of a number of complex psychological, social, technological and philosophical developments; it is not meant to be a potted history of memory. The point is to argue that the spread of use of technologies as memory supports has created a situation in which truth is normative for memory in ways that it was not, and could not have been, before those technologies existed, and that to treat truth as normative is to downplay other aspects of memory that could have been and no doubt were important in the evolution of the faculty in both non-human animals and human societies.

\section{Worries About Memory-Supporting Technology and Lifelogging}

The recent literature has thrown up some particular persistent worries about lifelogging, related to the perception that a person's lifelog contains truths that the human memory does not have, and that it is therefore reliable in a way that the unenhanced human is not. In particular, these are focused around the development of unbalanced, or psychologically disturbing, images, particularly self-images, and around the privacy of the individual. 
As an example of the first idea, legal scholar Anita Allen argues that an 'unredacted lifelog could turn into a bigger burden on balance' because 'electronic memory enables destructive reminding and remembrance' (Allen 2008, 56-57). We would be more prone to dredging up horrible memories from the past. 'The lifelogging concept is insensitive to the therapeutic value of forgetting the details of experience' (Allen 2010, 64). 'The technology will enable excessive rumination by persons experiencing unipolar or bipolar depression' (Allen 2010, 64-65). Political scientist Viktor Mayer-Schönberger agrees that the consequences of this technology are that stupid adolescent mistakes can take on disproportionate significance in later life (2009).

On privacy, Mayer-Schönberger also argues that 'comprehensive digital memory represents an even more pernicious version of the digital panopticon' so that 'the future has a chilling effect on what we do in the present' (MayerSchönberger 2009, 11-12). Allen sets out in some detail the argument that saving information about oneself would leave one open to invasions of privacy. Not only could one find oneself under surveillance (or, as it is sometimes termed, 'sousveillance') from lifelogger friends and acquaintances (Dodge \& Kitchin 2007, 434437), but also 'a government that has traditionally enjoyed access to communications and correspondence will want access to lifelogs' (Allen 2008, 67).

The purpose of this chapter is not to argue that these worries are unfounded. Quite the opposite; I am sympathetic, although I do think that they are often overstated. The danger, broadly, is that we will be confronted with the truth and nothing but the truth - but not necessarily (in fact, probably not) the whole truth.

The development of memory-supporting technology will result in a great deal of reliable information swilling around, relatively easy to access, from all sorts of sources including surveillance, sousveillance, social networking and lifelogging. Our social norms seem to be developing too slowly to keep pace; we live in a world of what we might call 'Intimacy 2.0', where rights to privacy are constantly neglected, eschewed, ignored or undervalued by a society that is increasingly exhibitionist and archival (O’Hara 2010b).

One danger of a situation where there is social upheaval while social norms fail to keep pace is that there will be pressure to conform; lifelogging is currently a fringe activity, and if all lifeloggers are volunteers then it may be unproblematic even if they become a majority. Allen anticipates the possibility that we might reach a situation where someone who wishes to retain control of the information about them (the traditional conception of informational privacy) comes to be seen to be abnormal; in that case, the fact that one does not keep a lifelog may itself be seen as suspicious (Allen 2008, 74). In such a world, our reasonable expectations of privacy (an important aspect of common-law protection of privacy) will decline (McArthur 2001, Bailey and Kerr 2007), with potentially deleterious effects across society.

There is an additional danger of seeing this sort of problem as exclusively a technological one. Not only could memory, which as Sellen and Whittaker argue $(2010,77)$ is a complex, multi-faceted set of concepts, come to be seen in an im- 
poverished way as what Proust called a 'simple cinematographic vision', but also that what may be sociotechnical problems come to be seen as amenable to technological solutions.

Entirely technical solutions are very unlikely to work. As has been noted in many quarters, the use of complex privacy controls merely confuses users; privacy-enhancing technologies generally suffer severe usability problems (Sasse and Flechais 2005). The point of lifelogging is that one does not have to think too hard about collecting, storing and retrieving information (O'Hara et al 2009); one of the ways that social networking sites like Facebook can get people to share information in more lucrative ways (for advertisers) is to set privacy defaults at a low level. Security techniques are similarly flawed; of course good security is a fine thing, but in a socio-technical system it is not just the technology but the way it is used that needs to be made secure. There is no point getting someone to create and regularly change a complex password if they end up having to resort to sticking it onto their computer screen with a Post-It (Inglesant and Sasse 2010).

\section{Mechanisms to Subvert the Record}

Hence a recent strand of thought has begun to develop the idea that the record itself could be subverted; this would have the effect of undermining the normative claims of truth. Mayer-Schönberger suggests the use of sell-by dates for information, so that stored information has associated with it a deletion date (MayerSchönberger 2009, 171-181). One creates one's Word file, say, and as part of the settings it might include a date when the file deletes itself (say, one year after the last edit). One could reset this at any time (as one can reset other metadata parameters, such as read and write permissions or filenames).

This idea has severe usability difficulties associated with it. The idea that one's old essays, letters or whatever might disappear because one forgot to set the delete-by date properly, is disturbing. It is hard to see it catching on; it seems a recipe for irritation (another box to tick before I can start editing my file), misunderstanding (particularly in a corporate context when files may have multiple editors with different ideas about this sort of thing), confusion (how does one calculate the time when information will become useless?), neglect (as one more and more often resorts to the default) and finally horror (oh my God my teenage novel/pictures of Grandpa/bookmarks relating to my old research have disappeared).

Dodge and Kitchin (2007) suggest that we might subvert the aims of those who wish to breach our privacy by a process of randomized falsification. Lifelogs might be programmed to change a small number of pieces of information so that they misrepresent reality. This is an interesting suggestion, as it uses the normativity of truth to undermine threats to privacy or self-perception; because truth is normative, and because it is possible that information retrieved from the lifelog is 
false, then the information, or what Bell calls the e-memory (Bell and Gemmell 2009), is that much less valuable.

This solution, though clever, is I think too clever by half. The problem is that although the normativity of truth is a problem, the value of the lifelog is its truth. Randomized falsification undoes some of the worries about memory-supporting technologies at the cost of rendering them less useful. In general, making them less useful will address all the worries given above, because if they are less useful they are less likely to be used, and therefore the anticipated problems with them are less likely to occur. The lifelog's creator wants access to information that is true; he is not interested in having false memories (the pro-lifelogging literature harps on at great length about the fallibility of memory - e.g. Bell and Gemmell 2009 , 51-56). So a system that serves up potentially false information seems not to fit the bill at all.

In general, philosophies of deletion and manipulation seem to throw the baby out with the bathwater; the advantages of abundant information seem clear and overwhelming, even if there will be associated difficulties. Information is clearly valuable, and is obviously perceived to be so because so many people spend so much time and effort trying to gather it. Storage and retrieval are incredibly cheap, certainly by historical standards, in which case the germane question is not 'why are we doing this?' but rather 'why not?'

\section{Conclusion: The Perils of Rich Representations}

Given the usefulness of writing, it seems that Socrates' plaints in the Phaedrus were overdone; few would advocate a return to an oral culture, even as an Edenic fantasy. However, his point is well-made in so far as the shift from orality to literacy required corresponding shifts in norms to regulate our expectations with respect to discourse in general. It may be, if lifelogging and the use of memorysupporting technologies take off as its advocates, like Bell, predict, that an analogous shift will also be required. We have been used to our pasts decaying from scrutiny at predictable rates; no doubt our e-memories will degrade, but not in a smooth way. One might lose last week's photographs while the ones of that embarrassing party thirty years ago remain stubbornly current. This is a new circumstance, where one's past cannot be expected simply to erase itself, and it is one to which we need to adapt. A past lifelog will have a presence, and we will need to understand what it is saying - and what it is not.

The point is not about good and bad technologies, but rather their use and misuse. We need to guard not against information processing and storage power, but rather what comes with them.

First of all, we need to guard against the closed world assumption. In computing and knowledge representation, this is the assumption that whatever cannot be asserted on the basis of a knowledge base is false - in other words, the assumption 
that the knowledge base is complete. With respect to a lifelog, or all lifelogs put together, or even the whole of the World Wide Web, this is a very dangerous assumption. To assume that 'if I can't find it with Google it can't be important' is extremely worrying in a world which is partially recorded by digital technologies, but where major inequalities of access correlated with age, educational achievement or nationality are evident.

Second, we must guard against the assumption of, or demand for, consistency. If truth is normative for memory, then inconsistency is symptomatic of a false memory somewhere. Yet given the shades of meaning and understanding underlying memories, it is not only plausible but commonplace to find different people with entirely different memories of an event, created and curated in good faith. A future world where one's testimony was automatically assessed as of less worth than, say, the records of one's Web browsing clickstream, or one's email inbox, or one's camera, would be a very worrying one. Even if truth remains normative for memory, the e-memories of browser, email program and camera are subject to interpretation too.

Third, we must guard against hindsight. Decisions made under uncertainty may seem to be poor, yet it is extremely easy to underestimate the complexity of realtime decision-making when we are in possession not only of the record of how the consequences of a decision unfolded, but also a richer picture of the context of that decision than could possibly have been available at the time.

Fourth, as many commentators have noted, there is an increasingly lack of interest in, and respect for, the distinction between public and private space. In part, this is the result of a lack of care in society as a whole, as I have argued elsewhere. One blatant misrepresentation that is often passed around is that privacy is in the interest of the individual, while publicity is in the interest of wider society ('the community'). Nothing could be further from the truth; abundant information and transparency are often in the interests of the individual, while privacy is in many respects a public good (O'Hara 2010b). Its neglect can often be seen as a tragedy of the commons (Anderson and Moore 2006).

Broadly speaking, our autonomy demands informational self-determination. That is not an easy thing to define or protect, and cannot simply be assimilated to our preferences for sacrificing privacy for material gain. In particular, even though the growth of lifelogging and memory-supporting technologies continues, we should be careful that this does not undermine our reasonable expectations of privacy. We should not be seduced by the richness of the lifelog into accepting all its assumptions, assertions and details.

We should, at all costs, retain the right to be a mystery. 


\section{Acknowledgements}

The work reported in this chapter was partly supported by the projects LiveMemories - Active Digital Memories of Collective Life, Bando Grandi Progetti 2006, Provincia Autonoma di Trento, and the EU FET project Living Knowledge, (http://livingknowledge-project.eu/), contract no. 231126. Thanks also to the audiences at the 2008 Workshop on Philosophy and Engineering at the Royal Academy of Engineering, and at the $2^{\text {nd }}$ Microsoft SenseCam workshop, held in Dublin, 2010 , when many of the ideas in this paper were presented in a keynote entitled Narcissus to a Man.

\section{References}

Allen, Anita L. 2008. Dredging up the past: lifelogging, memory, and surveillance. University of Chicago Law Review 75: 47-74.

Anderson, Ross and Tyler Moore. 2006. The economics of information security. Science 314: 610-613

Bailey, Jane and Ian Kerr. 2001. Seizing control? The experience capture experiments of Ringley and Mann. Ethics and Information Technology 9: 129-139.

Barry, Sebastian. 2008. The Secret Scripture. London: Faber \& Faber.

Bell, Gordon and Jim Gemmell. 2009. Total Recall: How the E-Memory Revolution Will Change Everything. New York: Dutton.

Berry, Emma, Narinder Kapur, Lyndsay Williams, Steve Hodges, Peter Watson, Gavin Smyth, James Srinivasan, Reg Smith, Barbara Wilson, Ken Wood. 2007. The use of a wearable camera, SenseCam, as a pictorial diary to improve autobiographical memory in a patient with limbic encephalitis: a preliminary report. Neuropsychological Rehabilitation 17: 582-601.

Byrne, Daragh, Aiden R. Doherty, Gareth J.F. Jones, Alan F. Smeaton, Sanna Kumpulainen and Kalervo Järvelin. The SenseCam as a tool for task observation. In People and Computers XXII: Culture, Creativity, Interaction: Proceedings of HCI 2008, 19-22. http://www.bcs.org/server.php?show=ConWebDoc.21389. Accessed 9 January 2011

De Bruijn, Oscar and Robert Spence. 2002. Rapid serial visual presentation. http://www.iis.ee.ic.ac.uk/ o.debruijn/rsvp.pdf. Accessed 9 January 2011.

Dix, Alan. 2002. The ultimate interface and the sums of life? Interfaces 50: 16.

Dodge, Martin and Rob Kitchin. 2007. 'Outlines of a world coming into existence': pervasive computing and the ethics of forgetting. Environment and Planning B: Planning and Design 34: 431-445.

Doherty, Aiden R., Cathal Gurrin and Alan F. Smeaton. 2009. An investigation into event decay from large personal media archives. $1^{\text {st }}$ ACM International Workshop on Events in Multimedia, Beijing, China. http://doras.dcu.ie/4722/1/eimm32412-doherty.pdf. Accessed 9 January 2011.

Garde-Hansen, Joanne, Andrew Hoskins and Anna Reading. 2009. Introduction. In Save As .... Digital Memories, ed. Joanne Garde-Hansen, Andrew Hoskins and Anna Reading, 1-26. Basingstoke: Palgrave Macmillan.

Goody, Jack. 1998. Memory in oral tradition. In Memory, ed. Patricia Fara and Karalyn Patterson, 73-94. Cambridge: Cambridge University Press. 
Hodges, Steve, Lyndsay Williams, Emma Berry, Shahram Izadi, James Srinivasan, Alex Butler, Gavin Smyth, Narinder Kapur and Ken Wood. 2006. SenseCam: a retrospective memory aid. In UbiComp 2006: Ubiquitous Computing, ed. Paul Dourish and Adrian Friday, 177-193. Berlin: Springer-Verlag.

Inglesant, Philip and M. Angela Sasse. 2010. The true cost of unusable password policies: password use in the wild. Proceedings of CHI 2010. http://hornbeam.cs.ucl.ac.uk/hcs/publications/Inglesant+Sasse The $\% 20$ True $\% 20$ Cost $\%$ 20of\%20Unusable\%20Password\%20Policies CHI2010.pdf. Accessed 13 January 2011.

Jimroglou, Krissi M. 1999. A camera with a view: JenniCAM, visual representation and cyborg subjectivity. Information, Communication and Society 2: 439-453.

Lee, Hyowon, Alan F. Smeaton, Noel O'Connor, Gareth Jones, Michael Blighe, Daragh Byrne, Aiden Doherty and Cathal Gurrin. 2008. Constructing a SenseCam visual diary as a media process. Multimedia Systems 14: 341-349. doi: 10.1007/s00530-008-0129-x.

Loftus, E.F. and J.C. Palmer. 1974. Reconstruction of automobile destruction: an example of the interaction between language and memory. Journal of Verbal Learning and Verbal Behaviour 13: 585-589.

Mayer-Schönberger, Viktor. 2009. Delete: The Virtue of Forgetting in the Digital Age. Princeton: Princeton University Press.

McArthur, Robert L. 2001. Reasonable expectations of privacy. Ethics and Information Technology 3: 123-128.

O'Hara, Kieron. 2010a. Arius in cyberspace: digital companions and the limits of the person. In Close Engagements With Artificial Companions: Key Social, Psychological, Ethical and Design Issues, ed. Yorick Wilks, 35-56. Amsterdam: John Benjamins.

O'Hara, Kieron. 2010b. Intimacy 2.0: privacy right and privacy responsibilities on the World Wide Web. Proceedings of the $2^{\text {nd }}$ Web Science Conference, Raleigh NC, http://journal.webscience.org/294/2/websci10 submission 3.pdf. Accessed 13 January 2011.

O'Hara, Kieron, Wendy Hall, Keith van Rijsbergen and Nigel Shadbolt. 2006a. Memory, reasoning and learning. In Cognitive Systems: Information Processing Meets Brain Science, ed. Richard Morris, Lionel Tarassenko and Michael Kenward, 236-260. Amsterdam: Elsevier.

O'Hara, Kieron, Richard Morris, Nigel Shadbolt, Graham J. Hitch, Wendy Hall and Neil Beagrie. 2006b. Memories for life: a review of the science and technology. Journal of the Royal Society Interface 3: 351-365. doi: 10.1098/rsif.2006.0125.

O'Hara, Kieron, Mischa M. Tuffield and Nigel Shadbolt. 2009. Lifelogging: privacy and empowerment with memories for life. Identity in the Information Society 1: 155-172. doi: 10.1007/s12394-009-0008-4

Ong, Walter. 1982. Orality and Literacy: The Technologizing of the Word. London: Methuen.

Plato. 1997. Phaedrus. In Complete Works, ed. John M. Cooper and D.S. Hutchinson, 507556

Sasse, M. Angela and Ivan Flechais. 2005. Usable security: Why do we need it? How do we get it? In Security and Usability: Designing Secure Systems That People Can Use, ed. Lorrie Faith Cranor and Simson Garfinkel, 13-30. Sebastopol CA: O'Reilly Media.

Sellen, Abigail, and Steve Whittaker. 2010. Beyond total capture: a constructive critique of lifelogging. Communications of the ACM 53(5): 70-77.

Shadbolt, Nigel. 2003. In memoriam. IEEE Intelligent Systems 18(6): 2-3. doi: 10.1109/MIS.2003.1200718.

Warnock, Mary. 1987. Memory. London: Faber \& Faber.

Wells, G.L. 1993. What do we know about eyewitness identification? Psychologist 48: 553571. 
Wilks, Yorick. 2010. Introducing artificial companions. In Close Engagements With Artificial Companions: Key Social, Psychological, Ethical and Design Issues, ed. Yorick Wilks, 11-20. Amsterdam: John Benjamins. 